\title{
Monitorização eletrocardiográfica ambulatorial por 24-horas em cães com cardiomiopatia dilatada idiopática
}

[Twenty-four-hour ambulatory electrocardiographic monitoring in dogs with idiopathic dilated cardiomyopathy]

\author{
F.L. Yamaki ${ }^{*}$ E.C. Soares, G.G. Pereira, V.M.C. Oliveira, M.H.M.A. Larsson \\ Faculdade de Medicina Veterinária e Zootecnia - USP \\ 05508-900 - São Paulo, SP
}

\begin{abstract}
RESUMO
Caracterizou-se monitorização eletrocardiográfica ambulatorial por 24 horas (ou monitorização Holter) em cães com cardiomiopatia dilatada idiopática, visando principalmente à detecção de arritmias ventriculares não detectadas pela eletrocardiografia convencional (de repouso). Para tanto, avaliaram-se 40 pacientes com diagnóstico de cardiomiopatia dilatada idiopática, por meio de exame físico e mensuração indireta da pressão arterial, além de exames eletrocardiográfico, ecocardiográfico, radiográfico de tórax e da monitorização Holter. Extra-sístoles ventriculares foram detectadas, por monitorização Holter, em $97,5 \%$ dos animais e taquicardia ventricular, em $45 \%$. Não houve correlação entre o número de extra-sístoles ventriculares e a fração de encurtamento. Considerando as manifestações clínicas, apenas houve associação entre presença de taquicardia ventricular e histórico de síncopes. Conclui-se que a incidência de arritmias ventriculares em cães com cardiomiopatia dilatada idiopática é bastante alta, sendo a taquicardia ventricular relativamente freqüente, ocorrendo mais sob a forma não sustentada.
\end{abstract}

Palavras-chave: cão, cardiomiopatia dilatada, Holter, eletrocardiografia, arritmia

\begin{abstract}
This study aimed to characterize 24-hour ambulatory electrocardiographic monitoring (Holter monitoring) in dogs with idiopathic dilated cardiomyopathy. Physical examination and indirect (Doppler) blood pressure measurement, and also electrocardiography, thoracic radiography, echocardiography, and 24-hour ambulatory electrocardiographic exams were performed in 40 dogs with idiopathic dilated cardiomyopathy. Ventricular extrasystoles were detected in $97.5 \%$ of the animals, and ventricular tachycardia in $45 \%$. No correlation between the number of ventricular extrasystoles and the shortening fraction was observed. Concerning the clinical symptoms, there was only association between the presence of ventricular tachycardia and past report of syncope. It was concluded that the incidence of ventricular arrhythmias is high in dogs with dilated cardiomyopathy, being ventricular tachycardia, mostly non-sustained, relatively frequent.
\end{abstract}

Keywords: dog, dilated cardiomyopathy, Holter, electrocardiography, arrhythmia

Recebido em 5 de outubro de 2006

Aceito em 3 de setembro de 2007

Endereço para correspondência (corresponding address)

R. Campos do Jordão, 200 apt. 32C - 05516-040 - São Paulo, SP

E-mail: fecardio@gmail.com

Apoio financeiro: FAPESP e CAPES 


\section{INTRODUÇÃO}

As cardiomiopatias (CMO) são atualmente definidas como doenças do miocárdio associadas com disfunção cardíaca, podendo ser classificadas nas formas: dilatada, hipertrófica, restritiva e arritmogênica do ventrículo direito; além disso, as $\mathrm{CMO}$ podem ser primárias (idiopáticas) ou secundárias - nas quais se conhece a causa de base (Richarson et al., 1996). Em cães, das CMO primárias ou idiopáticas, a dilatada é a mais prevalente, sendo uma das doenças cardiovasculares adquiridas mais comuns - apenas a doença valvar degenerativa e, em algumas regiões, a dirofilariose são causas mais importantes de morbidade e mortalidade cardíacas em cães (Sisson et al., 1999). Segundo Buchanan (1999), a cardiomiopatia dilatada (CMD) apresenta uma incidência de 11,3\% entre 300 cães cardiopatas atendidos no Hospital Veterinário da Universidade da Pensilvânia; ligeiramente menor que o encontrado por Larsson et al. (2000), no Brasil que relataram incidência de $15,8 \%$ entre 213 cães cardiopatas.

A CMD é uma síndrome caracterizada por alteração na função contrátil e dilatação do ventrículo esquerdo (VE) ou de ambos os ventrículos (Richardson et al., 1996). A anormalidade primária é a diminuição da contratilidade miocárdica, com dilatação secundária das câmaras cardíacas, havendo predomínio da disfunção sistólica, com hipertrofia miocárdica reacional nas áreas não acometidas pelo processo de agressão miocárdica; pode evoluir para insuficiência cardíaca (IC) ou não (dilatação sem IC), apresentar arritmias (atrial e/ou ventricular) e resultar em óbito em qualquer estágio da doença (Richardson et al., 1996; Sisson et al., 1999; Sisson et al., 2000; Meurs, 2005). A disfunção diastólica também pode ocorrer nos estágios avançados da doença, sendo menos evidente que a disfunção sistólica característica, além de ser de mais difícil diagnóstico (Sisson et al., 2000).

Apesar de ocorrer cada vez com maior freqüência em cães de raças médias como Dálmata, Cocker Spaniel Inglês e Americano, ainda é diagnosticada, mais comumente, em cães de raças grandes e gigantes como Boxer, Doberman, Dogue Alemão e São Bernardo (Sisson et al., 2000; Pereira et al, 2005).
Arritmias ventriculares e morte súbita (MS) são freqüentes em seres humanos (Dec e Valentin, 1991) e em cães, especialmente Dobermans (Calvert e Brown, 1986; Meurs, 2005) e Boxers (Harpster, 1991; Meurs, 2005) com cardiomiopatia dilatada. Em Dobermans, as arritmias ventriculares precedem evidências ecocardiográficas de CMD por alguns meses ou até anos (Dukes-McEwan et al., 2003); em Boxers, a deteç̧ão de arritmias ventriculares também precede o desenvolvimento de sintomatologia por três a quatro anos (DukesMcEwan et al., 2003). Infelizmente, o eletrocardiograma (ECG) realizado durante o exame físico tem limitada habilidade em detectar muitas arritmias, pois é realizado em repouso e por curto período de tempo (Miller et al., 1999; Cote e Ettinger, 2005). Dessa forma, o eletrocardiograma ambulatorial por longo período (ou monitorização Holter) consiste no teste não invasivo ideal para demonstrar e quantificar a freqüência e a complexidade de arritmias por longos períodos (Sisson et al., 1999; Nogueira et al., 2006).

Assim, a monitorização eletrocardiográfica ambulatorial (MEA) por 24 horas confirmou a ocorrência universal de extra-sístoles ventriculares (EV) e detectou taquicardia ventricular não sustentada (TVNS) em cerca de $40 \%$ dos pacientes humanos com CMD (Dec e Valentin, 1994); em cães, poucos são os trabalhos com MEA, sendo a maioria em Boxers e Dobermans. Desta forma, a verdadeira prevalência de arritmias na CMD ainda não foi bem estabelecida na veterinária, o que poderá ser mais bem definida com o aumento da utilização da monitorização Holter (DukesMcEwan et al., 2003).

O presente trabalho teve como objetivo a avaliação da monitorização Holter em cães com cardiomiopatia dilatada, principalmente em relação à quantificação e qualificação das arritmias ventriculares.

\section{MATERIAL E MÉTODOS}

Quarenta cães com diagnóstico de cardiomiopatia dilatada idiopática, baseado na diretriz proposta pela "força-tarefa ESVC para cardiomiopatia dilatada canina" (Dukes-McEvan et al., 2003), foram prospectivamente avaliados e incluídos no estudo - estudo Coorte. Os animais foram atendidos no Serviço de Cardiologia do Hospital Veterinário da Faculdade de Medicina Veterinária da Universidade de São Paulo, sendo avaliados por meio de exame físico, mensuração indireta (método de Doppler vascular) da pressão arterial, exames 
eletrocardiográfico convencional (de repouso), radiográfico de tórax, ecocardiográfico, além da monitorização Holter. Também foram realizados os seguintes exames laboratoriais: hemograma, perfil bioquímico (perfis renal e hepático, além de determinação de eletrólitos sódio e potássio). Por questões éticas todos os pacientes foram submetidos aos exames complementares na vigência de medicações que poderiam incluir diuréticos, digitálicos e inibidores da enzima conversora de angiotensina.

A gravação eletrocardiográfica ambulatorial foi realizada por 24 horas (monitorização Holter), com registro contínuo de três canais de ECG nas derivações pré-cordiais modificadas $\left(\mathrm{V}_{1}, \mathrm{~V}_{3} \mathrm{e}\right.$ $\mathrm{V}_{5}$ ), utilizando-se aparelho digital (marca DMS, modelo $300-7^{1}$ ), gravados em cartão eletromagnético (flash-card) e analisado por decodificação computadorizada (sistema cardio scan 10$)^{1}$. O gravador foi diretamente atado ao dorso do animal, oferecendo liberdade de movimento aos animais, com proteção do aparelho e dos cabos, que foram ligados a eletrodos adesivos (segundo descrição de Ware, 1998). Um diário, com as principais atividades do animal, foi realizado pelos proprietários.

A partir da monitorização Holter, determinou-se o ritmo de base, as freqüências cardíacas (FC) média, mínima e máxima e suas médias horárias, além de se quantificarem as arritmias supra e ventriculares. A qualificação das arritmias ventriculares foi realizada subdividindo-se os complexos em: isolados, pareados (duas EV consecutivas), bigeminismo ventricular (uma extra-sístole e um complexo normal), taquicardia ventricular (TV) não sustentada (TVNS) (série de três ou mais extra-sístoles ventriculares com freqüência maior que 160) e taquicardia ventricular sustentada (TVS) (com duração maior que 30 segundos); enquanto que a presença de três ou mais EV consecutivas com FC entre 70 e $160 \mathrm{bpm}$ foi denominada como ritmo idioventricular acelerado (RIA) (Côté e Ettinger, 2005).

As arritmias ventriculares também foram classificadas pelo método de Lown modificado (Meinertz at al., 1984): 0 - ausência de arritmias; 1 - complexos isolados e monomórficos com freqüência menor que 30/hora; 2 - complexos isolados e monomórficos com freqüência maior que 30/hora; 3 - complexos polimórficos; 4 complexos repetitivos (4 A - acopladas, $4 \mathrm{~B}$ TVNS).

Realizou-se análise estatística descritiva de cada uma das variáveis quantitativas, incluindo média, mediana e desvio-padrão (Berquó et al., 2002). As variáveis qualitativas foram comparadas pelo teste de qui-quadrado corrigido ou pelo teste exato de Fisher (nas situações em que os valores esperados foram inferiores a 5) (Siegel, 1977; Berquó et al., 2002). A distribuição normal (gaussiana) das variáveis quantitativas foi avaliada pelo teste de Kolmogorov-Smirnov, e os dados paramétricos (com distribuição normal e com homogeneidade das variâncias) foram comparados pelo teste t-Student (amostras independentes) ou teste t-Student pareado (amostras relacionadas) (Berquó et al., 2002), e os não paramétricos (sem distribuição normal e sem homogeneidade das variâncias) pelo teste $U$ de Mann-Whitney (amostras independentes) ou pelo teste de Wilcoxon (amostras relacionadas) (Siegel, 1977; Dawson e Trapp, 2003). Para descrever a correlação entre as variáveis paramétricas utilizou-se o coeficiente de correlação de Pearson e para as variáveis não paramétricas o coeficiente de Spearman (Siegel, 1977), além de se realizar teste de hipóteses para avaliar se o valor de $\mathrm{r}$ obtido era apenas devido à variabilidade das amostras ou se realmente existia correlação. Considerou-se como significante $\mathrm{P}$ menor que 0,05 .

\section{RESULTADOS}

A idade dos animais diagnosticados com CMD e incluídos no estudo variou entre dois e 15 anos, com média de 7,15 anos, mediana de 7,00 anos e desvio-padrão de 2,25 anos; não houve diferença significativa entre as idades dos machos e das fêmeas, no momento do diagnóstico $(\mathrm{P}=0,21)$. Dos 40 animais, 39 eram de raça pura e um animal era mestiço de duas raças predispostas (Doberman e Pastor Alemão); a maioria deles era de raça grande ou gigante (Fig. 1) e apenas oito, Cockers Spaniel, eram de porte médio. Dessa forma, o peso médio dos pacientes foi de $32,84 \mathrm{~kg}$, mediana de $32,70 \mathrm{~kg}$ e desvio padrão de $14,41 \mathrm{~kg}$ (variando de 10,6 a $74,2 \mathrm{~kg}$ ). Em relação ao sexo, houve predomínio de machos $(77,5 \%)$ em relação às fêmeas $(22,5 \%)$.

${ }^{1}$ DMS -São Paulo, Brasil. 


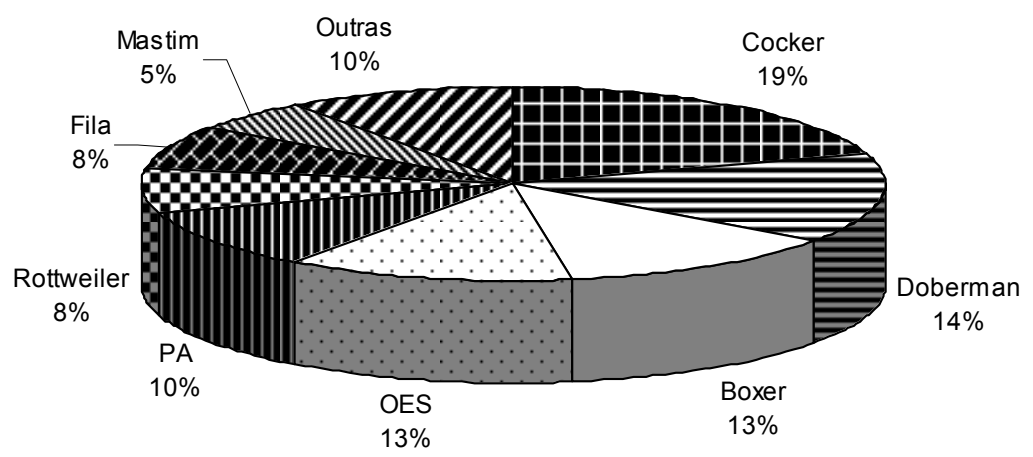

Cocker: Cocker Spaniel Inglês; OES: Old English Sheepdog; PA: Pastor Alemão; Fila: Fila Brasileiro; Mastim: Mastim Napolitado; Outras: outras raças.

Figura 1. Distribuição dos animais incluídos no estudo, segundo definição racial.

Em relação às manifestações clínicas, sete animais $(17,5 \%)$ apresentavam-se em CF-NYHA II (classe funcional da insuficência cardíaca segundo a New York Heart Association), 18 $(45,0 \%)$ em CF-NYHA III e 15 (37,5\%) em CFNYHA IV. Além disso, quatro animais (10,0\%) apresentavam histórico de síncope, 21 (52,5\%) estavam em edema pulmonar, nove $(22,5 \%)$ apresentavam efusão pleural, $18(45,0 \%)$ ascite e nove $(22,5 \%)$ edema de membros ou subcutâneo.

Quanto ao ritmo cardíaco, durante o exame eletrocardiográfico, o ritmo sinusal normal (ou arritmia sinusal) foi diagnosticado em 18 animais (45\%), a fibrilação atrial em 16 (40\%), a taquicardia sinusal em cinco $(12,5 \%)$ e a taquicardia atrial sustentada em um $(2,5 \%)$ paciente.

Em relação à monitorização Holter, alguns dados estão descritos na Tab. 1. Apenas um animal (Cocker Spaniel) não apresentou extra-sístoles ventriculares (EV) durante o Holter, sendo a taquicardia ventricular observada em $45 \%$ dos cães, chegando a ser sustentada em 12,5\% dos casos. Em outros 12,5\% dos casos, detectou-se a presença de ritmo idioventricular acelerado (RIA). Observaram-se, também, episódios de bigeminismo ventricular em $47,5 \%$ dos animais e pares em $72,5 \%$.

Entre os animais com TV na monitorização Holter, três eram da raça Fila Brasileiro, três da raça Boxer, três da raça Old English Sheepdog, três da raça Doberman, dois da raça Rottweiler, dois da raça Cocker Spaniel, um Mastim Napolitano e um Pastor Alemão. Considerando estes animais, o número de episódios de TV apresentados, durante a monitorização Holter, variou de 1 a 8.581 (com média de 902,06, mediana de 7,50 e DP de 2.440,50), com FC máxima na $\mathrm{TV}$, em média, de $226,39 \mathrm{bpm}$, com mediana de 229,00 e DP de 46,17 (variando de 161 a 292bpm); o episódio mais longo de cada animal variou de 3 a 284 complexos (com média de 63,00, mediana de 8,50 e DP de 97,55 complexos).

Tabela 1. Descrição das FC mínima, média e máxima, e do número de EV/24h e média de EV/h, obtidos por monitorização Holter em cães diagnosticados com cardiomiopatia dilatada idiopática

\begin{tabular}{lrrrrr}
\multicolumn{1}{c}{ Parâmetro } & \multicolumn{1}{c}{ Média } & \multicolumn{1}{c}{ Mediana } & \multicolumn{1}{c}{ DP } & \multicolumn{1}{c}{ Mínimo } & Máximo \\
\hline FC média $(\mathrm{bpm})$ & 130,05 & 128,50 & 26,76 & 82,00 & 184,00 \\
FC mínima $(\mathrm{bpm})$ & 57,35 & 55,00 & 20,31 & 31,00 & 111,00 \\
FC máxima $(\mathrm{bpm})$ & 213,43 & 213,00 & 22,35 & 158,00 & 254,00 \\
EV/24h & $6.704,60$ & 913,50 & $14.345,30$ & 0,00 & $75.141,00$ \\
EV/h & 288,60 & 42,50 & 607,09 & 0,00 & $3.131,00$ \\
\hline
\end{tabular}

DP: desvio-padrão; mínimo: valor mínimo obtido; máximo: valor máximo obtido; FC: freqüência cardíaca; bpm: batimentos por minuto; EV/24h: extra-sístoles por 24 horas; EV/h: média de extra-sístoles ventriculares por hora. 
Quanto à classificação de Lown modificada (Meinertz et al., 1984) das arritmias ventriculares, os dados estão dispostos na Fig. 2.

A correlação entre as $\mathrm{FC}$ obtidas em todos os pacientes pelo exame eletrocardiográfico convencional (Tab. 2) e pela monitorização Holter pelo coeficiente de Spearman foi positiva $\left(r_{\mathrm{s}}=0,488 ; \quad \mathrm{P}=0,0015\right), \quad$ sendo a $\mathrm{FC}$ significativamente maior durante o exame eletrocardiográfico de repouso $(\mathrm{P}=0,0002)$. Ao se considerarem apenas os animais com fibrilação atrial, a FC durante o ECG de repouso também foi significativamente mais alta do que durante a monitorização Holter $(\mathrm{P}<0,001)$, embora não tenha sido observada correlação significativa $\left(r_{p}=-0,0337 ; P=0,901\right)$ entre as $F C$ obtidas pela monitorização Holter e pela eletrocardiografia convencional.

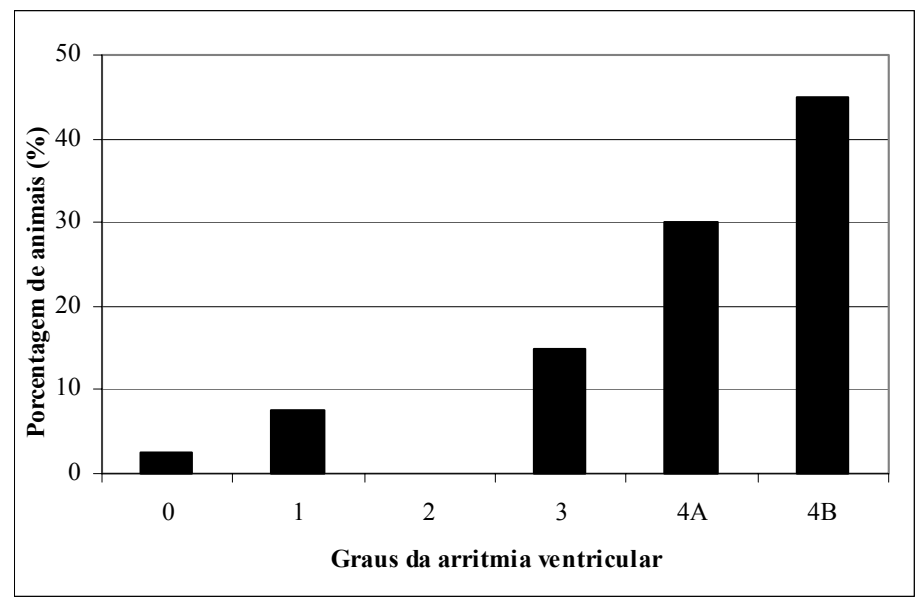

Figura 2. Distribuição dos animais diagnosticados com cardiomiopatia dilatada idiopática, segundo a classificação das arritmias ventriculares de Lown modificada (Meinertz at al., 1984).

Tabela 2. Descrição de alguns parâmetros eletrocardiográficos obtidos pela eletrocardiografia convencional e por monitorização Holter dos cães diagnosticados com cardiomiopatia dilatada idiopática

\begin{tabular}{|c|c|c|c|c|}
\hline Parâmetro & \multicolumn{2}{|c|}{ ECG convencional } & \multicolumn{2}{|c|}{ Holter } \\
\hline \multicolumn{5}{|l|}{ Freqüência cardíaca (bpm) } \\
\hline Média & \multicolumn{2}{|c|}{167,49} & \multicolumn{2}{|c|}{130,05} \\
\hline Mediana & \multicolumn{2}{|c|}{160,00} & \multicolumn{2}{|c|}{128,50} \\
\hline Desvio-padrão & \multicolumn{2}{|c|}{49,97} & \multicolumn{2}{|c|}{26,76} \\
\hline Arritmias & $\mathrm{N}$ & $\%$ & $\mathrm{~N}$ & $\%$ \\
\hline Fibrilação atrial & 16 & 40,0 & 16 & 40,0 \\
\hline Taquicardia atrial & 1 & 2,5 & 15 & 62,5 \\
\hline Extra-sístole ventricular & 11 & 27,5 & 39 & 97,5 \\
\hline Taquicardia ventricular & 2 & 5,0 & 18 & 45,0 \\
\hline
\end{tabular}

ECG: eletrocardiograma; bpm: batimentos por minuto; N: número de animais.

Dentre os 39 animais com arritmias ventriculares na monitorização Holter, apenas 28,2\% também apresentaram EV durante o ECG convencional (com duração menor que um minuto).

Utilizando-se o coeficiente de correlação de Spearman, observou-se correlação positiva entre o número de EV/24 horas e o número de taquicardias ventriculares $\left(\mathrm{r}_{\mathrm{s}}=0,634, \mathrm{P}<0,001\right)$, o número de bigeminismos ventriculares $\left(\mathrm{r}_{\mathrm{s}}=0,641\right.$; $\mathrm{P}<0,001)$ e o número de pares de $\mathrm{EV}\left(\mathrm{r}_{\mathrm{s}}=0,857\right.$; $\mathrm{P}<0,001)$. Não houve correlação entre o número de $E V / 24-h$ e a FC média $\left(r_{s}=0,020 ; P=0,901\right)$, mínima $\left(\mathrm{r}_{\mathrm{s}}=-0,034 ; \mathrm{P}=0,837\right)$ ou máxima $\left(\mathrm{r}_{\mathrm{s}}=-\right.$ $0,101 ; \mathrm{P}=0,537)$.

Não houve correlação entre o número de EV obtido na monitorização Holter e a fração de 
encurtamento obtida pelo exame ecocardiográfico $\left(\mathrm{r}_{\mathrm{s}}=0,162 ; \mathrm{P}=0,324\right)$.

Não se encontrou associação entre a presença de taquicardia ventricular e a CF-NYHA $(\mathrm{P}=0,984)$, nem entre a presença de TV e de FA $(\mathrm{P}=0,399)$, de edema pulmonar $(\mathrm{P}=0,356)$, de efusão pleural $(\mathrm{P}=0,705)$, de ascite $(\mathrm{P}=0,565)$ e de edema subcutâneo $(\mathrm{P}=0,149)$. Houve associação, estatisticamente significativa, entre o histórico de síncope e a presença de TV no Holter $(\mathrm{P}=0,033)$. Em relação aos quatro animais com histórico de síncope (dois Boxers, um Doberman e uma Cocker Spaniel), nenhum apresentou o episódio sincopal durante a monitorização, mas todos tiveram pelo menos um episódio de taquicardia ventricular na monitorização Holter, incriminando-se, assim, a arritmia ventricular como causa do sintoma. Considerando-se somente os animais com histórico de síncope, o número médio de TV/24-h foi de 2148 (variando de 1 a 8581) episódios, com duração de 7,5 complexos (variação de três a 11) e com FC máxima na TV de 234,25bpm (variação de 180 a 274). Todos os animais com síncope apresentaram morte súbita, apesar da instituição da terapia antiarrítmica.

Deve-se ressaltar, também, que nenhum animal apresentava hipopotassemia no momento de sua inclusão no estudo.

\section{DISCUSSÃO}

Dos 40 animais incluídos no estudo, 77,5\% eram machos, prevalência sexual que está em acordo com o que é descrito na literatura para cães (Monnet et al., 1995; Tidholm, et al., 1997; Sisson et al., 1999) e em seres humanos (Huang et al., 1983; Meinetz et al., 1984; Grimm et al., 2005). A idade dos animais (com média de 8,10 anos) também foi semelhante àquela relatada na literatura, embora ligeiramente mais alta que a média de sete anos obtida por Monnet et al. (1995) e de 6,6 anos obtida por Tidholm et al. (1997).

As raças mais freqüentemente acometidas foram Cocker Spaniel, Doberman, Boxer e Old English Sheepdog, que são tradicionalmente reconhecidas como portadoras da doença (Harpster, 1991; Tidholm et al., 1997; Monnet et al., 1995; Sisson et al., 2000). Embora, em relação à predisposição racial, não se possa esquecer que a incidência da CMD varia entre as diferentes raças, havendo variações geográficas numa mesma raça (Sisson et al., 2000).

Todos os registros da monitorização Holter contaram com mais de 20 horas de duração. Optou-se por se realizar a colocação do gravador no hospital veterinário, sendo $\mathrm{o}$ paciente dispensado e permanecendo, a maior parte do período de 24 horas, em seu ambiente domiciliar.

A FC média, obtida pela monitorização Holter $(130,05 \mathrm{bpm})$, foi significativamente mais baixa do que a obtida pela eletrocardiografia convencional $(160,00 \mathrm{bpm})$, realizada no ambiente hospitalar, o que pode ser explicado pela estimulação simpática ocasionada por tal situação estressante ao animal. Tal fato também foi notado por Miller et al. (1999) ao realizarem a monitorização Holter em cães com histórico de síncopes em relação ao eletrocardiograma convencional no hospital veterinário (respectivamente, 93bpm X 124bpm). O mesmo ocorreu em relação à $\mathrm{FC}$ dos animais com fibrilação atrial (143,81bpm X 210,33bpm); dessa forma, o registro Holter também é o mais indicado para monitorar a $\mathrm{FC}$ em cães com fibrilação atrial (FA), que pode estar artificialmente elevada nas visitas ao clínico veterinário, conforme citado por Gelzer e Kraus (2004). Ao se considerar os animais com FA, também não se pode esquecer que eles estavam sob efeito da terapia com digoxina, que apresenta eficácia apenas parcial no controle da FC, sendo inadequada sob estresse e excitação (Gelzer e Kraus, 2004), o que pode explicar a falta de correlação entre as FC obtidas pela ECG convencional e pela monitorização Holter.

A baixa sensibilidade da ECG convencional em detectar arritmias transitórias já havia sido observada por Meurs et al. (2001), em cães Boxers, e por Lipski et al. (1976), em pacientes humanos com síncopes; embora, no presente estudo, a sensibilidade tenha sido mais baixa $(28,21 \%)$ do que a observada por Meurs et al. (2001) (variando entre $36 \%$ e $92 \%$ ) e por Lipski et al. (1976) (de 54,54\%). Meurs et al. (2001) notaram, também, que a sensibilidade variava com a freqüência de ocorrência na monitorização Holter (sendo mais alta quanto maior o número de EV). De qualquer forma, tal dado corrobora a afirmação de Dukes-McEwan et al. (2003) de que a incidência das arritmias ventriculares é 
mais bem definida com a utilização da monitorização Holter.

Apenas um animal não apresentou EV durante a monitorização Holter, confirmando a ocorrência praticamente universal das arritmias ventriculares em cães com cardiomiopatia dilatada, similar à incidência de 100\% encontrada por Calvert e Brown (1986) em Dobermans (utilizando exame electrocardiográfico convencional e monitorização Holter em alguns animais) e mais alta que a incidência de 45,5\% em Boxers (Harpster, 1991) e de $27 \%$ em cães de várias raças (obtida por Monnet et al., 1995 em estudo retrospectivo) por meio de exame eletrocardiográfico convencional.

A TV foi detectada em $45 \%$ dos cães, freqüência semelhante à observada em seres humanos por Meinertz et al. (1984) e Huang et al. (1983) respectivamente, $49 \%$ e $60 \%$, em cães Boxers por Harspster (1991), 38,4\%, e em cães Dobermans por Calvert et al. (1997), 38\%. Entretanto, deve-se ressaltar que o estudo de Harpster (1991) em Boxers não utilizou a monitorização Holter.

Contrariamente ao observado por Huang et al. (1983), que notaram que os pacientes humanos com TV eram mais velhos, no presente estudo não houve correlação entre idade e presença de $\mathrm{TV}$, semelhantemente aos achados de Grimm et al. (2005), também em seres humanos com CMD.

Da mesma forma que em humanos com CMD (Huang et al., 1983; Meinertz et al., 1984), não se observou correlação entre a gravidade da arritmia ventricular e a gravidade da insuficiência cardíaca ou da disfunção ventricular. Embora Grimm et al., (2005), ao estudarem pacientes humanos com CMD, tenham observado relação entre a presença de TVNS e pior CF-NYHA e menor fração de ejeção. Tal divergência de resultados pode ser explicada pelo tamanho da amostra, que foi bem maior no estudo de Grimm et al. (2005), com 343 pacientes incluídos, ao passo que Huang et al. (1983) apenas incluíram 35 pacientes e Meinertz et al. (1984) 74 pacientes.

$\mathrm{Na}$ contagem das EV, excluíram-se os complexos ventriculares de escape. Quanto ao
RIA, arritmia com menor comprometimento hemodinâmico e mais bem tolerada (Côte e Ettinger, 2005), Grimm et al. (2000) não encontraram correlação com menor sobrevida em seres humanos com CMD, concluindo que são achados eletrocardiográficos benignos. Desta forma, embora o significado do RIA ainda não tenha sido estabelecido na cardiologia veterinária, ele foi excluído da análise das arritmias ventriculares.

\section{CONCLUSÃO}

A incidência de arritmias ventriculares em cães com cardiomiopatia dilatada é bastante alta, sendo a taquicardia ventricular relativamente freqüente, ocorrendo mais sob a forma não sustentada. Além disso, há associação entre histórico de síncopes e presença de taquicardia ventricular na monitorização Holter.

\section{REFERÊNCIAS BIBLIOGRÁFICAS}

BERQUÓ, E.S.; SOUZA, S.M.P.; GOTLIEB, S.L.D. Bioestatística. 2.ed. São Paulo: Ed. Pedagógica e Universitária Ltda., 2002. 349p.

BUCHANAN, J.W. Prevalence of cardiovascular disorders. In: FOX, P.R.; SISSON, D.; MOISE, N.S. Textbook of canine and feline cardiology. 2.ed. Philadelphia: W.B. Sanders, 1999. p.457-470.

CALVERT, C.A.; BROWN, J. Use of M-mode echocardiography in the diagnosis of congestive cardiomyopathy in Doberman pinschers. J. Am Vet. Med. Assoc. v.189, p.293-297, 1986.

CALVERT, C.A.; HALL, G.; JACOBS, G. et al. Clinical and pathologic findings in Doberman Pinschers with occult cardiomyopathy that died suddenly or developed congestive heart failure: 54 cases (1984 - 1991). J. Am. Vet. Med. Assoc., v.210, p.505-511, 1997.

CÔTÉ, E.; ETTINGER, S.J. Electrocardiography and cardiac arrhythmias. In: ETTINGER, S.J.; FELDMAN, E.C. (Eds). Textbook of veterinary internal medicine. 6.ed. St. Louis: Elsevier Saunders, 2005. p.1040-1076.

DAWSON, B.; TRAPP. R.G. Bioestatística básica e clínica. 3.ed. Rio de Janeiro: McGraw Hill, 2003. $348 \mathrm{p}$.

DEC, G.W.; VALENTIN, F. Medical progress: idiopathic dilated cardiomyopathy. N. Engl. J. Med., v.331, p.1564-1575, 1994. 
DUKES-McEWAN, J.; BORGARELLI, M.; TIDHOLM, A. et al. Proposed guidelines for the diagnosis of canine idiopathic dilated cardiomyopathy - The ESVC Taskforce for Canine Dilated Cardiomyopathy. J. Vet. Cardiol., v.5, p.7-18, 2003.

GELZER, A.R.M.; KRAUS, M.S. Management of atrial fibrillation. Vet. Clin. N. Am. Small Anim. Pract., v.34, p.1127-1144, 2004.

GRIMM, W.; CHRIST, M.; MAISCH, B. Long runs of non-sustained ventricular tachycardia on 24-hour ambulatory electrocardiogram predict major arrhythmic events in patients with idiopathic dilated cardiomyopathy. Pacing Clin. Eletrophysiol., v.28, suppl.1, p.s207-s210, 2005

GRIMM, W.; HOFFMANN, et al. Significance of accelerated idioventricular rhythm in idiopathic dilated cardiomyopathy. Am. J. Cardiol., v.85, p.899-904, 2000 .

HARPSTER, N.K. Boxer cardiomyopathy - a review of the long-term benefits of antiarrhythmic therapy. Vet. Clin. N. Am. Sm. Anim. Pract., v.21, p.989-1004, 1991.

HUANG, S.K.; MESSER, J.V.; DENES, P. Significance of ventricular tachycardia in idiopathic dilated cardiomyopathy: observations in 35 patientes. Am. J. Cardiol., v.51, p.507-512, 1983.

LARSSON, M.H.M.A.; BARBUSCI, L.O.D.; SOARES, E.C. et al. Estudo ecocadiográfico das cardiopatias mais freqüentemente diagnosticadas em espécimes caninos. In: CONGRESSO BRASILEIRO DE CLÍNICOS VETERINÁRIOS, 21., 2000, Rio de Janeiro. Rev. Bras. Ciênc. Vet., v.7, supl., p.68-68, 2000.

LIPSKI, J.; COHEN, L.; ESPINOZA, J. Value of Holter monitoring in assessing cardiac arrhythmias in symptomatic patients. Am. J. Cardiol., v.37, p.102107, 1976.

MEINERTZ, T.; HOFMANN, T.; KASPER, W.; et al. Significance of ventricular arrhythmias in idiopathic dilated cardiomyopathy. Am. J. Cardiol., v.53, p.902907, 1984.

MEURS, K.M. Primary myocardial disease in the dog. In: ETTINGER, S.J.; FELDMAN, E.C. (Eds). Textbook of veterinary internal medicine. 6.ed. St. Louis: Elsevier Saunders, 2005. p.1077-1082.

MEURS, K.M.; SPIER, A.W.; WRIGHT, N.A. et al., Comparison of in-hospital versus 24-hour ambulatory electrocardiography for detection of ventricular premature complexes in mature Boxers. J. Am. Vet. Med. Assoc., v.218, p.222-224, 2001.

MILLER, R.H.; LEHMKUHL, L.B.; BONAGURA, J.D. et al. Retrospective analysis of the clinical utility of ambulatory electrocardiographic (Holter) recordings in syncopal dogs: 44 cases $(1991-1995)$. $J$. Vet. Intern. Med., v.13, p.111-122, 1999.

MONNET, E.; ORTON, C.O.; SALMAN, M.; BOON, J. Idiopathic dilated cardiomyopathy in dogs: survival and prognostic indicators. J. Vet. Intern. Med., v.9, p.12-17, 1995.

NOGUEIRA, R.B.; MUZZI, R.A.L.; HERRERA, D.S. et al. Avaliação do ritmo cardíaco em cães da raça Boxer saudáveis pela eletrocardiografia contínua (Holter). Arq. Bras. Med. Vet. Zootec., v.58, p. 133136, 2006.

PEREIRA, P.M.; CAMACHO, A.A.; MORAIS, H.A. Tratamento de insuficiência cardíaca com benazepril em cães com cardiomiopatia dilatada e endocardiose. Arq. Bras. Med. Vet. Zootec., v.57, supl.2, p.141-148, 2005.

RICHARDSON, P.; MACKENNA, W.; BRISTOW, M. et al. Report of the 1995 World Health Organization - International Society and Federation of Cardiology task force on definition and classification of cardiomyopathies. Circulation, v.93, p.841-842, 1996.

SIEGEL, S. Estatística não-paramétrica para as ciências do comportamento. 1.ed. São Paulo: McGraw-Hill do Brasil Ltda, 1977. 349p.

SISSON, D.; O'GRADY, M.R.; CALVERT, C.A. Myocardial diseases of dogs. In: FOX, P.R.; SISSON, D.; MOISE, N.S. Textbook of canine and feline cardiology. 2.ed. Philadelphia: W.B. Sanders, 1999. p.581-619.

SISSON, D.; THOMAS, W.P.; KEENE, B.W. Primary myocardial disease in the dog. In: ETTINGER, S.J.; FELDMAN, E.C. Textbook of veterinary internal medicine: diseases of the dog and cat. 5.ed. Philadelphia: W.B. Saunders, 2000. p.874895.

TIDHOLM, A.; JONSSON, L.; SYLVÉN, C. A retrospective study of canine dilated cardiomyopathy (189)] cases. J. Am. Anim. Hosp. Assoc., v.33, p.544$550,1997$.

WARE, W. Practical use of Holter monitoring. Comp. Cont. Educ. Pract. Vet., v.20, p.167-177, 1998. 